\title{
THE RADIUS OF GYRATION OF A CONVEX BODY ${ }^{1}$
}

\section{E. J. MCSHANE}

The purpose of this note is to establish an inequality of isoperimetric type for convex bodies. Let $K$ be a bounded convex body and $l$ a line through its centroid; let $\delta(K, l)$ be the supremum of distances of points of $K$ from $l, I(K, l)$ the moment of inertia of $K$ about $l$, and $g(K, l)$ the radius of gyration $[I(K, l) / \text { mass } K]^{1 / 2}$ of $K$ about $l$. If $K$ were not restricted to be convex, it is easy to show that the values of the ratio $g(K, l) / \delta(K, l)$ consist of all numbers in the open interval $(0,1)$. However, under the restriction to convex $K$ we shall show that the infimum of this ratio is $1 / 15^{1 / 2}$. The usual methods of the calculus of variations are unavailable, as is usual in problems concerning convex bodies.

If $K$ is a bounded convex body in three-space, and $l$ is a line in three-space, and $\delta(x, y, z ; l)$ the distance from the point $(x, y, z)$ to $l$, we define the mass, $m(K)$, centroid $(\bar{x}(K), \bar{y}(K), \bar{z}(K))$, moment of inertia $I(K, l)$ and radius of gyration $g(K, l)$ as usual:

$$
\begin{aligned}
m(K) & =\int_{K} d x d y d z, \quad \bar{x}(K)=\int_{K} x d x d y d z / m(K), \text { etc., } \\
I(K, l) & =\int_{K} \delta(x, y, z ; l)^{2} d x d y d z, \quad g(K, l)=[I(K, l) / m(K)]^{1 / 2} ;
\end{aligned}
$$

and we define

$$
\delta(K, l)=\sup \{\delta(x, y, z ; l):(x, y, z) \epsilon K\} .
$$

It is obvious that none of these quantities change if we replace $K$ by its closure. Likewise, if a linear mass-distribution in an interval $[a, b]$ is defined by a density $A(x)(a \leqq x \leqq b)$, and $c$ is a real number, we define

$$
\begin{array}{rlrl}
m(A) & =\int_{a}^{b} A(x) d x, & \bar{x}(A) & =\int_{a}^{b} x A(x) d x / m(A), \\
I(A, c) & =\int_{a}^{b}(x-c)^{2} A(x) d x, & g(A, c) & =[I(A, c) / m(A)]^{1 / 2} \\
\delta(A, c) & =\text { larger of }|a-c|,|b-c| .
\end{array}
$$

Received by the editors August 28, 1961. G-63.

1 This work was supported by the U. S. Army Research Office Grant No. DA-ARO- 
We simplify the notation when $c=\bar{x}(A)$, writing

(4) $\quad I(A)=I(A, \bar{x}(A)), \quad g(A)=g(A, \bar{x}(A)), \quad \delta(A)=\delta(A, \bar{x}(A))$.

TheOREM. If $K$ is a bounded convex body and $l$ a line through its centroid, and $\delta(K, l)$ is the supremum of distances of points of $K$ from $l$ and $g(K, l)$ the radius of gyration of $K$ about $l$, then

$$
g(K, l) / \delta(K, l)>1 / 15^{1 / 2} .
$$

In this statement the constant $1 / 15^{1 / 2}$ cannot be replaced by any larger number.

By the remark after (2), we may and shall restrict our attention to closed convex bodies. Given any $K$ and $l$ as in the theorem, we choose rectangular axes so that $l$ is the $z$-axis and the positive $x$-axis contains a point at distance $\delta(K, l)$ from $l$. The projection of $K$ on the $x$-axis is an interval $[a, b]$ with $-\delta(K, l) \leqq a<b=\delta(K, l)$. For each $\xi$ in $[a, b]$ let $A(\xi)$ be the area of the intersection $K(\xi)$ of $K$ with the plane $x=\xi$. By the Brunn-Minkowski theorem $\left[1\right.$, p. 88] $[A(x)]^{1 / 2}(a \leqq x \leqq b)$ is a concave function, obviously continuous in the open interval $(a, b)$ and by the closure of $K$ continuous at $a$ and $b$ also. Also, $\bar{x}(A)=\bar{x}(K)=0$, so $\delta(A)=b=\delta(K, l)$. For the moments of inertia we have

$$
\begin{aligned}
I(K, l) & =\int_{a}^{b}\left\{\int_{K(x)}\left(x^{2}+y^{2}\right) d y d z\right\} d x \\
& >\int_{a}^{b} \int_{K(x)} x^{2} d y d z d x \\
& =\int_{a}^{b} x^{2} A(x) d x=I(A),
\end{aligned}
$$

so

$$
g(A) / \delta(A)<g(K, l) / \delta(K, l) .
$$

Let $\mathcal{F}$ be the family of functions $A$ each defined, continuous and non-negative on some closed interval and having $[A]^{1 / 2}$ concave on that interval. The function $A$ of the preceding paragraph belongs to F. So if we define

$\mu=$ infimum of $g(K, l) / \delta(K, l)$ for all convex bodies $K$ and all lines through the centroid of $K$,

$\mu^{\prime}=$ infimum of $g(A) / \delta(A)$ for all $A$ in $\mathcal{F}$,

by (6) we have 


$$
\mu^{\prime} \leqq \mu
$$

On the other hand, let $A(x)(a \leqq x \leqq b)$ belong to $F$. For each positive $\epsilon$ let $K$. be the solid of revolution obtained by revolving the set $\left\{(x, y): a \leqq x \leqq b, 0 \leqq y \leqq \epsilon[A(x) / \pi]^{1 / 2}\right\}$ about the $x$-axis. Then $K_{\text {c is }}$ convex, and its centroid is $(\bar{x}(A), 0,0)$. Let $l$ be the line $x=\bar{x}(A)$, $y=0$. It is easy to see that as $\epsilon$ tends to $0, \delta\left(K_{\epsilon}, l\right)$ tends to $\delta(A)$. Also,

$$
\begin{aligned}
I\left(K_{\epsilon}, l\right) & =\int_{a}^{b}\left[(x-\bar{x}(A))^{2}+\epsilon^{2} A(x) / 4 \pi\right] \epsilon^{2} A(x) d x, \\
m\left(K_{\epsilon}\right) & =\int_{a}^{b} \epsilon^{2} A(x) d x,
\end{aligned}
$$

so

$$
\lim _{\epsilon \rightarrow 0} g\left(K_{\epsilon}, l\right)=\left[\int_{a}^{b}(x-\bar{x}(A))^{2} A(x) d x / \int_{a}^{b} A(x) d x\right]^{1 / 2}=g(A) .
$$

Therefore as $\epsilon$ tends to $0, g\left(K_{\epsilon}, l\right) / \delta\left(K_{\epsilon}, l\right)$ tends to $g(A) / \delta(A)$, and $\mu$ cannot be greater than $\mu^{\prime}$. This and (8) prove that

$$
\mu=\mu^{\prime} \text {, }
$$

and (9) and (6) prove that for all $K$ and $l$ as in the theorem, $g(K, l) / \delta(K, l)>\mu$. It remains to prove that $\mu=1 / 15^{1 / 2}$. The function $A(x)=(1-x)^{2}(0 \leqq x \leqq 1)$ belongs to $\mathcal{F}$, and we readily compute that for it

$$
g(A) / \delta(A)=1 / \sqrt{ } 15
$$

Hence

$$
\mu \leqq 1 / \sqrt{ } 15 .
$$

Suppose now that $\mu<1 / 15^{1 / 2}$. Let $A_{1}, A_{2}, \cdots$ be a sequence of functions belonging to $\mathfrak{F}$ such that

$$
\lim _{n \rightarrow \infty} g\left(A_{n}\right) / \delta\left(A_{n}\right)=\mu .
$$

The ratio $g(A) / \delta(A)$ is unaltered by transformations $x \rightarrow h x+k$ with $h>0$, so we may suppose that all the $A_{n}$ have $[0,1]$ as domain. Also, the ratio is unaffected by multiplication of $A$ by a positive constant, so we may suppose that all $A_{n}$ have supremum 1 . Then for each positive in teger $k$ all the functions $A_{n}$ satisfy the Lipschitz condition with constant $1 / k$ on the interval $[1 / k, 1-1 / k]$, so we may select a subsequence uniformly convergent on that interval. By the diagonal process we obtain a subsequence converging to a limit $A(x)$ for each $x$ in $(0,1)$. Then $[A]^{1 / 2}$ is concave, so $A(x)$ has limits as $x \rightarrow 0+$ and 
as $x \rightarrow 1-$. We assign $A(0)$ and $A(1)$ these respective values. Then $A$ belongs to $\mathcal{F}$ and satisfies

$$
g(A) / \delta(A)=\mu .
$$

Since multiplication by a positive constant leaves $A$ in $\mathcal{F}$ and leaves the left member of (12) unchanged, we may assume $m(A)=1$. Likewise, by applying the transformation $x \rightarrow 1-x$ if necessary, we may obtain $\bar{x}(A) \leqq 1 / 2$. From $A$ we construct $K_{\mathbf{c}}$ by rotation of $\epsilon[A / \pi]^{1 / 2}$, as before; this is convex and its centroid is $(\bar{x}(A), 0,0)$, so $[1, \mathrm{p} .52]$

$$
1 / 4 \leqq \bar{x}(A) \leqq 1 / 2 \text {. }
$$

To simplify notation we shall define

$$
c=\bar{x}(A) \text {. }
$$

Let $\epsilon$ be any positive number less than 1 ; the $O$ and $o$ notation will be used for estimates with $\epsilon$ near 0 . Define

$$
\alpha=\int_{0}^{e} A(x) d x
$$

then $\alpha=0(\epsilon)$. Let $A$, be the restriction of $A$ to the subinterval $[\epsilon, 1]$. Then

$$
\begin{aligned}
m\left(A_{\epsilon}\right) & =1-\alpha, \\
m\left(A_{\epsilon}\right) \bar{x}\left(A_{\epsilon}\right) & =\int_{\bullet}^{1} x A(x) d x=c+\alpha O(\epsilon),
\end{aligned}
$$

whence

$$
\bar{x}\left(A_{\epsilon}\right)=c(1+\alpha)+\alpha O(\epsilon) .
$$

Also

$$
\begin{aligned}
I\left(A_{\epsilon}\right) & =I\left(A_{\epsilon}, c\right)-\left[\bar{x}\left(A_{\epsilon}\right)-c\right]^{2} m\left(A_{\epsilon}\right) \\
& =I(A)-\int_{0}^{\epsilon}(c-x)^{2} A(x) d x+O\left(\alpha^{2}\right) \\
& =I(A)-c^{2} \alpha[1+O(\epsilon)],
\end{aligned}
$$

whence

$$
g\left(A_{\epsilon}\right)^{2}=I(A)\left\{1+\alpha\left[1-c^{2} / I(A)+O(\epsilon)\right]\right\} .
$$

Since

$$
\delta\left(A_{\epsilon}\right) \geqq 1-\bar{x}\left(A_{\epsilon}\right)=\delta(A)-c \alpha+\alpha O(\epsilon),
$$

we have 


$$
\begin{aligned}
& {\left[g\left(A_{\epsilon}\right) / \delta\left(A_{\epsilon}\right)\right]^{2}} \\
& \quad \leqq I(A) / \delta(A)^{2}\left\{1+\alpha\left[1-c^{2} / I(A)+2 c / \delta(A)+O(\epsilon)\right]\right\} .
\end{aligned}
$$

By definition of $A$, with (12),

$$
I(A) / \delta(A)^{2}=[g(A) / \delta(A)]^{2}=\mu^{2}<1 / 15,
$$

so, (allowing the next equation to define $C$ )

$$
\begin{aligned}
C & =1-c^{2} / I(A)+2 c / \delta(A)=1-c^{2} / \mu^{2} \delta(A)^{2}+2 c / \delta(A) \\
& =-\left(\frac{c}{\mu \delta(A)}-\mu\right)^{2}+\mu^{2}+1 .
\end{aligned}
$$

Since $c / \delta(A)=c /(1-c)$, which is increasing on $[0,1)$, and $1 / 4 \leqq c$ $\leqq 1 / 2$, we have $c / \delta(A) \geqq 1 / 3$. Since $\mu^{2}<1 / 15$, we also have $c / \mu \delta(A)$ $-\mu>0$, and the right member of (17) is not decreased if we replace $c / \delta(A)$ by $1 / 3$ :

$$
C \leqq 1+\mu^{2}-\left(\frac{1}{3 \mu}-\mu\right)^{2} .
$$

The derivative with respect to $t$ of the function $\phi(t)=(1 /(3 t)-t)^{2}$ is

$$
\phi^{\prime}(t)=-\frac{2}{t}\left(\frac{1}{9 t^{2}}-t^{2}\right)
$$

which is negative for $t$ in $(0,1 / 3)$. Hence the right member of (18) is increased if we replace $\mu$ by the larger number $1 / 15^{1 / 2}$, so that

$$
C<1+1 / 15-\left(15^{1 / 2} / 3-1 / 15^{1 / 2}\right)^{2}=0 .
$$

The coefficient $C-O(\epsilon)$ of $\alpha$ in (15) is therefore negative for all positive $\epsilon$ near 0 , and $\alpha$ is positive, so for all such $\epsilon$ we have by (15) and (16)

$$
g\left(A_{\epsilon}\right) / \delta\left(A_{\epsilon}\right)<\mu .
$$

But this contradicts the definition of $\mu$, and the proof is complete.

If $K$ is a right circular cylinder with axis $l$, it is easily computed that $g(K, l) / \delta(K, l)=1 / 2^{1 / 2}$. I conjecture, but have not been able to complete a proof, that $1 / 2^{1 / 2}$ is in fact the supremum of the ratio $g(K, l) / \delta(K, l)$ for all convex bodies $K$ and all lines $l$ through the centroid of $K$.

\section{BIBLIOGRAPHY}

1. T. Bonnesen and W. Fenchel, Theorie der konvexen Körper, Springer, Berlin, 1934.

UNIVERSITY OF VIRGINIA 